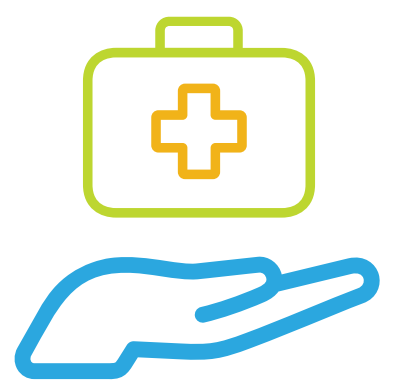

\title{
A EXPERIÊNCIA DO APOIO PARA O FORTALECIMENTO DO MANEJO DA SÍFILIS NA ATENÇÃO PRIMÁRIA À SAÚDE
}

\author{
THE EXPERIENCE OF SUPPORT FOR STRENGTHENING THE MANAGEMENT OF \\ SYPHILIS IN PRIMARY HEALTH CARE
}

\begin{abstract}
Luis Saulo Sousa Santos
Enfermeiro especialista em Educação para a Saúde (UFRGS), especialista em Saúde Coletiva com Ênfase no Trabalho Interprofissional em Saúde (FIOCRUZ/UFMA), Apoiador de pesquisa e intervenção Projeto Sífilis Não (UFRN/MS). E-mail: drsauloribeiro@live.com

Thamyris Mendes Gomes Machado

Enfermeira especialista em Saúde da Família e Comunidade (UESPI), Mestranda do Mestrado Profissional em Saúde da Família, PROFSAÚDE (FIOCRUZ/UFMA), Integrante do Programa Força Estadual de Saúde do

Maranhão. E-mail: thamyris-bioenff@hotmail.com.

Silvia Cristina Viana Silva Lima
\end{abstract}

Enfermeira, docente da UFMA e técnica do Departamento de IST/HIV/Aids e Hepatites Virais da SES-MA. E-mail: silvia.viana@ufma.br

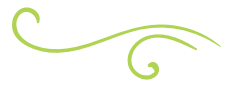

\section{RESUMO}

Este estudo apresenta uma experiência de intervenção vivenciada junto aos profissionais da Atenção Primária das Unidades Básicas de Saúde de um município da região metropolitana do Maranhão, executada no contexto do Projeto Resposta Rápida à Sífilis "Sífilis Não" do Ministério da Saúde, durante o mês de outubro de 2019. Utilizou uma metodologia que envolveu a educação permanente em saúde, com estabelecimento de vários espaços coletivos com participações efetivas. A intervenção aconteceu em 04 etapas, com várias estratégias de diálogos, trocas, debates e estudos de casos sobre a sífilis adquirida e congênita. O primeiro momento consistiu em uma reunião para pactuações e alinhamentos da proposta com a Secretaria Municipal de Saúde e Secretaria de Estado da Saúde; na segunda etapa, oficializou a intervenção e houve a comunicação aos gerentes das Unidades Básicas de Saúde; a etapa três contou com a realização das rodas de conversas in loco nas Unidades Básicas de Saúde, durante três semanas, envolvendo 22 equipes de Estratégia Saúde da Família, perfazendo um total de 271 participantes. $O$ estudo observou a dificuldade por parte de alguns profissionais, médicos e enfermeiros, na condução do manejo da sífilis no pré-natal das gestantes e suas parcerias sexuais, considerando a oferta e a solicitação do exame, o diagnóstico de sífilis na gestante, o tratamento e a importância do Venereal Disease Research Laboratory para o seguimento dos 
casos. A pesquisa evidenciou que muitas condutas não estavam em consonância com o Protocolo Clínico e Diretrizes Terapêuticas para Atenção Integral às Pessoas com Infecções Sexualmente Transmissíveis (2019), do Ministério da Saúde, que estabelece os critérios para diagnóstico de infecções/ doenças ou agravos à saúde, o tratamento, com recomendações a ser seguidas pelos profissionais de saúde e gestores do Sistema Único de Saúde. Quanto aos odontólogos, estes necessitam ser envolvidos nas ações de prevenção e manejo da sífilis. A etapa final permitiu análises sobre o nível de conhecimento adquirido pelos profissionais, e revelou a importância do apoio institucional junto ao Projeto "Sífilis Não". O estudo considera que a intervenção contribuiu para a reorganização das práticas colaborativas desses profissionais da atenção primária à saúde, na perspectiva de melhoria na atenção aos(às) usuários(as) com sífilis e o incentivo a participarem de outras ações, intervenções e estudos sobre a temática.

Palavras-chave: Sífilis; Gestação; Educação permanente em Saúde.

\section{ABSTRACT}

An intervention experience lived with Primary Care professionals from Basic Health Units (UBS) of a municipality in the metropolitan region of Maranhão, presented in the context of the Rapid Response to Syphilis No Syphilis Project of the Ministry of Health (MS), is presented., during the month of October 2019. A methodology that involved permanent health education was used, with the establishment of several collective spaces with effective participation. The intervention took place in four stages, with various strategies for dialogues, exchanges, debates and case studies on acquired and congenital syphilis. The first moment consisted of a meeting to agree and align the proposal with Municipal Health Secretariat and State Health Secretariat; in the second stage, the intervention was made official and there was communication to the managers of the UBS; stage three consisted of carrying out conversations on the spot in the UBS for three weeks, involving 22 teams from the Family Health Strategy, making a total of 271 participants. It was observed the difficulty on the part of some professionals, doctors and nurses, in conducting the management of syphilis in the prenatal care of pregnant women and their sexual partnerships, related since the offer and request for the exam, the diagnosis of syphilis in the pregnant woman, the treatment and the importance of the Venereal Disease Research Laboratory for the follow-up of cases. It became evident that many conducts were not in line with the Clinical Protocol and Therapeutic Guidelines for Comprehensive Care for People with Sexually Transmitted Infections (2019), from the MS, which establishes the criteria for the diagnosis of infections / diseases or health problems, the treatment, with recommendations to be followed by health professionals and managers SUS. And that dentists need to be involved in the prevention and management of syphilis. The final stage allowed for analyzes of the level of knowledge acquired by the professionals, and revealed the importance of institutional support with the Syphilis Project No. It is considered that the intervention contributed to the reorganization of the collaborative practices of these professionals in primary health care, in the perspective improvement in care for users with syphilis and the incentive to participate in other actions, intervention and studies on the subject.

Key-words: Syphilis; Pregnancy; Health Education.

\section{INTRODUÇÃO}

Parte-se da concepção de apoio proposta por Campos (2005) como método de intervenção que incide sobre as relações de poderes e de saberes presentes nas 
instituições, visando a um coletivo crítico que possa produzir análises sobre essas relações e assumir compromissos conjuntos. Nesse contexto, compreende-se que a tarefa do apoiador consiste em acompanhar os grupos e os auxiliar a instaurar processos de cogestão que permitam transformar os processos de trabalho. $\bigcirc$ apoio é função institucional e metodologia de intervenção, ativa em espaços coletivos que propiciam a interação e a construção conjunta entre os sujeitos, reconhecendo e manejando, com os afetos envolvidos, as relações de poder e a multiplicidade de saberes para a construção de objetivos comuns, pactuações e contratos que promovem a capacidade de análise crítica dos coletivos (MACERATA; SOARES; OLIVEIRA, 2019).

É preciso, portanto, incorporar o ensinar e o aprender aos processos cotidianos de trabalho e aos "problemas/fazeres/saberes" que surgem e dinamizam os modos de gerir e produzir saúde. Ações de formação devem, portanto, ser inserias e funcionar no cotidiano dos serviços e redes de saúde. Isso significa, na multiplicidade do território e na complexidade da atenção primária, colocar a formação "à disposição do exercício de apreender com e no mundo do trabaIho, enquanto um campo essencialmente micropolítico". O processo de formação nos convida a vivenciar "o cuidar, durante o ato do trabalho" (COÊLHO; MIRANDA; NETO, 2019). Nesse cenário desafiador, torna-se relevante trabalhar ações de educação permanente em saúde com os profissionais, o que permite vivências e contribuições dos sujeitos envolvidos, fortalecendo processos autônomos dos sujeitos e grupos sociais que acarretam mudanças nas práticas de saúde da comunidade (AZEVEDO et al., 2014).

Em 2018, o Ministério da Saúde implantou o Projeto Interfederativo de Resposta Rápida à Sífilis nas Redes de Atenção, com o objetivo de diminuir a sífilis adquirida, sífilis em gestantes, e erradicar a sífilis congênita no Brasil por meio de 4 eixos: fortalecimento da vigilância epidemiológica; gestão e governança; cuidado integral; educação em saúde e comunicação. Buscou-se, dessa forma, assegurar ações integradas e colaborativas com articulação dos diferentes pontos de atenção à saúde, setores sociais e comunidade (BRASIL, 2019).

Ademais, as informações do Boletim Epidemiológico do Ministério da Saúde (2019) mostram que a sífilis adquirida, agravo de notificação compulsória desde 2010, teve sua taxa de detecção aumentada de 59,1 casos por 100.000 habitantes, em 2017, para 75,8 casos por 100.000 habitantes, em 2018. Também em 2018, a taxa de detecção de sífilis em gestantes foi de 21,4/1.000 nascidos vivos, a taxa de incidência de sífilis congênita foi de 9,0/1.000 nascidos vivos e taxa de mortalidade por sífilis congênita foi de 8,2/100.000 nascidos vivos.

Como sabemos, a sífilis é uma infecção bacteriana sistêmica, crônica, curável e exclusiva do ser humano. Quando não tratada, evolui para estágios de gravidade variada, podendo acometer diversos órgãos e sistemas do corpo. Trata-se de uma infecção conhecida há séculos. Seu agente etiológico, descoberto em 1905, é o Treponema pallidum, subespécie pallidum. Sua transmissão se dá principalmente por contato sexual; contudo, pode ser transmitida verticalmente para o feto, durante a gestação de uma mulher com sífilis não tratada ou tratada de forma não adequada (BRASIL, 2019). A sífilis congênita (SC) é o resultado da disseminação/transmissão hematogênica do Treponema pallidum da gestante infectada não tratada ou inadequadamente tratada para o concepto por via transplacentária, podendo ser transmitida em qualquer fase gestacional ou em qualquer estágio da doença materna (BRASIL, 2019).

Tal situação demonstra que, apesar dos esforços para prevenção e controle, o número de casos de sífilis e sífilis congênita no Brasil e no Maranhão continua a crescer. Entendendo que a sífilis deveria ser uma infecção erradicada - e não está -, conseguimos visualizar a fragmentação do cuidado no 
percurso do (a) usuário (a) com sífilis na linha de cuidado. Determina-se, então, a necessidade de analisar e investir na prevenção dos casos a partir da descentralização das ações, fortalecendo o diagnóstico, o tratamento e o seguimento. Além disso, torna-se imprescindível a reorganização da linha de cuidado, com base em uma abordagem com práticas colaborativas e envolvimento dos diferentes dispositivos de saúde (SILVA, 2014).

Este artigo objetiva relatar a experiência de apoio institucional com intervenções realizadas junto aos profissionais da atenção primária das unidades básicas de saúde de um município da região metropolitana do Maranhão. A relevância dessa intervenção contribuiu diretamente para a reorganização das práticas colaborativas dos profissionais da atenção primária à saúde, na perspectiva de que seus resultados possibilitaram o fortalecimento das ações e, ainda, vislumbrando melhoria na atenção aos usuários (as) com sífilis e o incentivo para outros estudos aprofundados sobre a temática.

\section{MÉTODO}

Trata-se de um relato de experiência, vivenciado durante a execução do Projeto Pesquisa Aplicada para Integração Inteligente Orientada ao Fortalecimento das Redes de Atenção para Resposta Rápida à Sífilis uma parceria entre o Laboratório de Inovação Tecnológica em Saúde (LAIS), o Ministério da Saúde (MS) e a Organização Pan-Americana da Saúde (OPAS), desenvolvido com os profissionais da Atenção Primária em Saúde (APS) durante o mês de outubro de 2019. Os relatos de experiência são tidos como metodologias de observação sistemática da realidade, sem o objetivo de testar hipóteses, mas estabelecendo relações entre os achados dessa realidade e bases teóricas pertinentes (DYNIEWICZ, 2014).

A intervenção foi realizada em 04 etapas. $\bigcirc$ primeiro momento constituiu em uma reunião de pactuações para alinhamento da proposta com representantes da Atenção Primária em Saúde e Vigilância em Saúde (VS) da Secretaria Municipal de Saúde com apoio do Departamento de IST/HIV/Aids e Hepatites Virais da Secretaria de Estado da Saúde, oportunidade em que houve a explanação da proposta, pelo apoiador das ações, que seria desenvolvida com os profissionais.

$\mathrm{Na}$ segunda etapa, houve a comunicação oficial/institucionalizada aos gerentes das Unidades Básicas de Saúde (UBS) com informação sobre o objetivo da intervenção, a data e horário de realização na UBS. Na ocasião, foi destacada a importância da participação de todos os profissionais das equipes de saúde da família (médicos, enfermeiros, dentistas, técnicos de enfermagem e agentes comunitários de saúde).

Na terceira e quarta etapa, objetivou-se a validação da pactuação e avaliação da intervenção. Foram desenvolvidas/executas as rodas de conversas, com duração de três semanas, envolvendo os profissionais de saúde das 16 Unidades Básicas de Saúde (UBS) desse município da região metropolitana do Maranhão. Para a realização da intervenção, foram agendados previamente os encontros do apoiador com os profissionais, por intermédio da Coordenação Municipal das Infecções Sexualmente Transmissíveis (IST), Aids e Hepatites Virais e dos gerentes das unidades.

\section{RESULTADOS}

Durante a intervenção, especificamente nas rodas de conversa realizadas in loco nas UBS, durante três semanas, conforme Quadro 1, foram abordados temas referentes à sífilis, tais como: manejo da sífilis na atenção primária, sífilis em gestantes e suas parcerias sexuais, administração da penicilina na UBS e notificação compulsória. Alcançaram-se 16 unidades de saúde e 22 equipes de Estratégia Saúde da Família (ESF), perfazendo um total de 271 profissionais participantes, de diferentes categoriais. 


\begin{tabular}{|c|c|c|c|}
\hline ORDEM & SERVIÇO DE SAÚDE & QUANTIDADE DE ESF & DATA \\
\hline 01 & UBS Paranã & 02 & 07/10/2019 \\
\hline 02 & UBS Maiobão & 03 & 07/10/2019 \\
\hline 03 & UBS Itapera Mocajituba & 01 & 08/10/2019 \\
\hline 04 & UBS da Pirâmide & 01 & 08/10/2019 \\
\hline 05 & UBS Santa Rita de Cassia & 01 & 09/10/2019 \\
\hline 06 & UBS Vila Cafeteira & 01 & 09/10/2019 \\
\hline 07 & UBS Tambaú & 02 & $14 / 10 / 2019$ \\
\hline 08 & UBS Safira & 01 & $14 / 10 / 2019$ \\
\hline 09 & UBS Maioba & 02 & $15 / 10 / 2019$ \\
\hline 10 & UBS da Pindoba & 01 & $15 / 10 / 2019$ \\
\hline 11 & UBS Vila São José & 02 & $16 / 10 / 2019$ \\
\hline 12 & UBS do Canaã & 01 & $16 / 10 / 2019$ \\
\hline 13 & $\begin{array}{l}\text { UBS Nossa Senhora da } \\
\text { Luz }\end{array}$ & 01 & $21 / 10 / 2019$ \\
\hline 14 & UBS Mojó & 01 & $21 / 10 / 2019$ \\
\hline 15 & UBS Iguaíba & 01 & $21 / 10 / 2019$ \\
\hline 16 & UBS Vila São Pedro & 01 & $22 / 10 / 2019$ \\
\hline
\end{tabular}

Quadro 1 - Cronograma da Roda de Conversa nas Unidades Básicas de Saúde. Município selecionado. MA. Fonte: autoria própria (2019).

A intervenção do apoio institucional realizou-se com as 22 Equipes de Saúde da Família, permitindo-se questionamentos pelos participantes, além da utilização de fluxogramas, discograma e material impresso que subsidiaram o diálogo e contribuíram para o processo de aprendizado, na perspectiva das práticas colaborativas. Durante todo o processo, observaram-se dificuldades de alguns profissionais médicos e enfermeiros na condução do manejo da sífilis no pré-natal com as gestantes e suas parcerias sexuais. Ocorrem relatos que evidenciaram condutas que não estavam em consonância com o Protocolo Clínico e Diretrizes Terapêuticas para Atenção Integral às Pessoas com Infecções Sexualmente Transmissíveis (PCDT IST) (2019), do Ministério da Saúde, que estabelece os critérios para diagnóstico de infecções/doenças ou agravos à saúde, o tratamento preconizado com medicamentos e demais produtos apropriados, as posologias recomendadas, os mecanismos de controle clínico, o acompanhamento e a verificação dos resultados terapêuticos a ser seguidos pelos profissionais de saúde e gestores do Sistema Único de Saúde (SUS).

Assim, percebeu-se e foi constatado, junto aos profissionais médicos e enfermeiros, dúvidas em diferentes âmbitos: desde a oferta e solicitação do exame treponêmico e não treponêmico para sífilis, até fechar o diagnóstico de sífilis na gestante, prescrição e administração da penicilina benzatina para o tratamento e a importância do VDRL para o seguimento dos casos. Chamou-nos a atenção, durante as rodas de conversas, que profissionais dentistas relataram que era 0 primeiro contato com o assunto. Afirmaram a importância da avaliação odontológica 
para auxiliar no diagnóstico da sífilis na fase primária da infecção, o que contribuirá com a equipe para o devido diagnóstico.

Compreende-se, portanto, que a estratégia mais concreta de alcançar a prevenção e o controle da sífilis está no compromisso da APS em realizar uma abordagem com as gestantes e suas parcerias sexuais. Essa atenção inclui um pré-natal, de qualidade, garantindo o diagnóstico precoce e o tratamento adequado para evitar a transmissão vertical da infecção. A esse respeito, Lima et al. (2013) ressaltam o curto intervalo da gestação para a realização do seu diagnóstico e tratamento; a dificuldade de abordagem das doenças sexualmente transmissíveis, principalmente durante a gestação; e, provavelmente, o desconhecimento da magnitude desse agravo e dos danos que ele poderá causar à saúde da mulher e do bebê pela população e pelos profissionais de saúde.

Na realização da rodada de perguntas, a partir de estudo de casos, que aconteceu de forma aleatória, foram abordados aspectos múltiplos de prevenção, diagnóstico, tratamento e seguimento dos casos de sífilis. Foi solicitado a cada profissional que proferisse uma palavra para avaliar o momento com o intuito de construir uma nuvem de palavras. As palavras mais citadas: aprendizado, enriquecimento, contribuição profissional, gratidão, desafiador, conhecimento, o que demonstra a necessidade de educação permanente em saúde.

Nesse contexto, fica evidente que também a Educação em Saúde possibilita uma prática social, cujo processo contribui para a formação da consciência crítica das pessoas a respeito de seus problemas de saúde, a partir da sua realidade, e estimula a busca de soluções e organização para a ação individual e coletiva (FEUERWERKER, 2007). Dessa forma, a etapa final da intervenção permitiu discussões e análises sobre o nível de conhecimento adquirido pelos profissionais, o que revelou que o apoio institucional favorece práticas colaborativas, visto que a maior parte das perguntas foi respondida corretamente, demonstrando que o objetivo da intervenção foi alcançado, contando com a participação ativa de todos os participantes, e envolveu dirigentes, coordenadores e gestores de diferentes níveis de atuação.

\section{CONSIDERAÇÕES FINAIS}

A vivência, a partir da intervenção realizada, permitiu constatar que esse momento de educação permanente em saúde proporcionou um aumento do conhecimento sobre o manejo da sífilis com ênfase na conduta do pré-natal das gestantes e suas parcerias sexuais, na perspectiva de mudanças nos processos de trabalho.

É importante destacar que o apoio institucional favorece e possibilita as ações de educação em saúde junto aos profissionais para que se qualifiquem e realizem, em conjunto com os serviços de saúde, o manejo adequado dos casos de sífilis. Essa ação buscar melhorar a prevenção e o controle da infecção na comunidade, garantindo, assim, - fortalecimento das Unidades Básicas de Saúde que devem estar preparadas para identificar os casos, possibilitando o diagnóstico precoce e o tratamento oportuno.

Nessa experiência, foi possível perceber a multiplicidade de espaços e públicos que foram envolvidos nas intervenções educativas dessa natureza, além de ter proporcionado um conhecimento abrangente de fatores determinantes e condicionantes do processo saúde-doença/cuidado da comunidade local. Ademais, mostrou aos profissionais da APS, gerentes de unidades, coordenação e apoiador a importância da integração da Vigilância em Saúde com a Atenção Primária para o fortalecimento do manejo da sífilis nos territórios vivos. Por fim, aponta-se como estratégia para superar as dificuldades encontradas a possibilidade de articular ações intersetoriais entre as instituições e os serviços de saúde do município, com a finalidade de proporcionar subsídios, como recursos materiais e 
humanos qualificados para a condução de um planejamento racional e efetivo num território determinado, especialmente em microáreas da Estratégia Saúde da Família.

\section{AGRADECIMENTOS}

À coordenação e supervisão do Projeto "Sífilis Não".

Ao Departamento de Atenção às IST, Aids e Hepatites Virais da Secretaria de Estado da Saúde, por toda acolhida e pelo apoio às ações territoriais.

À Secretaria Municipal de Saúde de Paço do Lumiar.

A Lúcia Nayara Leite de Melo, Coordenadora Municipal de IST, por toda a dedicação e o comprometimento durante a realização das oficinas.

Aos profissionais médicos, enfermeiros, dentistas, técnicos de enfermagem e agentes comunitários de saúde, pela disponibilidade e pelo compromisso com as oficinas.

Às apoiadoras, Chyrly Elidiane de Moura e Vânia Priamo, pela condução nas atividades assíncronas neste cenário de pandemia da covid-19.

A Eduardo Carvalho, pela motivação. 


\section{REFERÊNCIAS}

AZEVEDO, I. C. et al. Compartilhando saberes através da Educação em Saúde na Escola: Interfaces do Estágio Supervisionado em Enfermagem. REnfermCentOMin, v. 04, n.1, p. 1048-1056, jan./abr. 2014.

BRASIL. Ministério da Saúde. Protocolo Clínico e Diretrizes Terapêuticas para Atenção Integral às Pessoas com Infecções Sexualmente Transmissíveis (IST). Brasília: Ministério da Saúde, 2019.

BRASIL. Ministério da Saúde. Boletim Epidemiológico de Sífilis. Brasília, ano V, n. 1, 2019. Disponível em: http://www.aids.gov.br/pt-br/pub/2019/boletim-epidemiologico-sifilis-2019. Acesso em: 10 fev. 2020.

CAMPOS, G. W. Um método para análise e cogestão de coletivos: a constituição do sujeito, a produção de valor de uso e a democracia em instituições: o método da roda. 2. ed. São Paulo: Hucitec, 2005.

COÊLHO, B. P.; MIRANDA, G. M. D.; NETO, O. B. C. A Formação-Intervenção na Atenção Primária: uma Aposta Pedagógica na Educação Médica. Revbraseducmed, Brasília, v. 43, n.1, supl.1, maio 2019.

DYNIEWICZ, A. M. Metodologia da pesquisa em saúde para iniciantes. 3. ed. São Caetano do Sul: Difusão, 2014.

FEUERWERKER, L. C. M. Educação na saúde - educação dos profissionais de saúde - um campo de saber e de práticas sociais em construção. Revbraseducmed, Rio de Janeiro, v. 31, n.1, p.39, jan./abr. 2007.

LIMA, G. K. et al. Educação em Saúde sobre Sífilis com um grupo de gestantes: um relato de experiência de acadêmicas de enfermagem. SANARE, Sobral, v. 12, n. 2, p. 59-62, jun./ dez. 2013.

MACERATA, I; SOARES, J.G.N.; OLIVEIRA, A. M. A pesquisa-intervenção como pesquisaapoio: o caso do POP RUA. Saudesoc, São Paulo, v. 28, n.4, out./dez. 2019.

SILVA, J. L. Fragilidades e desafios na consolidação da linha de cuidado do usuário com sífilis em Santa Maria/RS. 2014. Trabalho de Conclusão de Curso (Pós-graduação em Residência Multiprofissional Integrada em Sistema Público de Saúde) - Universidade Federal de Santa Maria, Santa Maria, 2014. Disponível em: https://repositorio.ufsm.br/ bitstream/handle/1/487/Silva_Juliana_Lima_da.pdf?sequence=1. Acesso em: 23 nov. 2020. 